\title{
Pt(IV)Ac-P0A: New Platinum Compound Induced Caspase Independent Apoptosis In B50 Neuroblastoma Stem Cells
}

\author{
Ferrari B1, Camuso S1, Priori EC1, De Luca F1, Roda E1,3, Osella D $^{2}$ \\ and Bottone $\mathrm{MG}^{1 *}$ \\ ${ }^{1}$ Department of Biology and Biotechnology, University of Pavia, Italy \\ 22Department of Sciences and Technological Innovation (DiSIT)"University of Eastern \\ Piedmont, Italy \\ ${ }^{3}$ Laboratory of Clinical \& Experimental Toxicology, Pavia Poison Centre, National \\ Toxicology Information Centre, Toxicology Unit, ICS Maugeri Spa, IRCCS Pavia, Italy
}

\begin{tabular}{|c|}
\hline Research Article \\
Volume 3 Issue 2 \\
Received Date: August 17, 2019 \\
Published Date: September 06, 2019 \\
DoI: $10.23880 /$ jes-16000125 \\
\hline
\end{tabular}

*Corresponding author: Maria Grazia Bottone, Department of Biology and Biotechnology, University of Pavia, Via Ferrata 9, 27100, Pavia, Italy, Tel: 3388527987; Email: bottone@unipv.it

\section{Abstract}

Neuroblastoma is a tumour that affects adults and children, characterized by a stem cells component. To date, cisplatin is the main antitumor agent used in the clinical treatment of this tumour; however, it induces side effects such as neurotoxicity in healthy cells and induces chemo resistance to therapy in cancer cells. New platinum-based compounds, platinum (II) have recently been synthesized, and due to their chemical characteristics, they are able to identify new cellular targets. These complexes act as prodrugs and performing their cytotoxic effect as platinum (II) after a reduction reaction within the hypoxic tumour cells. Among these prodrugs, Pt(IV)Ac-POA appears to be very promising, thanks to the presence of ligand (2-propinyl)octanoic acid (POA), which acts as an inhibitor of histone deacetylase (HDACi) and leads to the increase of histone acetylation, decreasing the interactions between histone and DNA, so as to produce chemo-sensitization to DNA-damaging agents. The greater cytotoxic effect of Pt(IV)Ac-POA on tumour cells, would therefore be mainly due to the mechanism of inhibition of histone deacetylase, which would increase the accessibility of DNA to platination mechanisms that induce cell death. In this study the results show that Pt(IV)Ac-POA, used at a concentration ten times lower than cisplatin, can induce apoptosis in B50 cells in culture both through the intrinsic pathway and through the independent caspase pathway. The data, obtained by immunohistochemical techniques in fluorescence microscopy, show that treatment with Pt(IV)Ac-POA has a greater proapoptotic effect on stem cells compared to the cisplatin standard treatment.

Keywords: B50 Cells; Apoptosis; Cisplatin; Pt(IV)Ac-POA 


\section{Journal of Embryology \& Stem Cell Research}

\section{Introduction}

Cisplatin is one of the most active chemotherapeutic drug used in the treatment of different type of solid cancer, including some nervous system tumours, such as Neuroblastoma [1]. Neuroblastoma is a frequently extracranial tumour in infancy, representing $8-10 \%$ of all cancer in childhood. To date, cisplatin is largely employed in Neuroblastoma treatment, but despite its benefit, its used is hampered by severe systemic side effects and the onset of drug resistance [2-4]. For this reason, in the last years the goal of biomedical research has been the synthesis of new antitumor agents, with the same antitumor effect of the reference drug, but associated to less systemic toxicity.

To overcome limits of classical oncotherapy, new platinum (IV) pro drugs have been synthesised. The Pt(IV) complexes act as pro drugs and reducing themselves to cytotoxic analogues of Pt(II) inside hypoxic tumour cells [5-7]. Among these, the new Pt(IV)Ac-POA pro drug represents a promising tool, bearing as axial ligand (2-propynyl) octanoic acid (POA), an histone deacetylase inhibitor (HDACi), Pt(IV)Ac-POA is able to produce a synergistic action in the hypoxic tumour cell microenvironment. Indeed, HDAC inhibition increases histone acetylation, decreasing histone-DNA interactions and allowing for chemo-sensitization versus DNAdamaging agents [8-10]. The new prodrug has showed also a higher activity due to the high cellular accumulation by virtue of high lipophilicity and to the inhibition of histone deacetylase which leads to increased exposure of nuclear DNA, thereby permitting higher platination levels and promoting cancer cells death.

In this study we evaluated the effect of the new Pt(IV)Ac-POA on the B50 Neuroblastoma rat cells, focusing on the activation of different cell death pathways and the morphological and functional changes inducted by treatment.
The B50 neuronal cell line offer several advantages to the study of neurons in culture, in fact they are simple to grow, to differentiate and to transfect. Concerning to our research, B50 cells have been extensively used in the study of death and toxicology.

\section{Materials and Methods}

B50 Neuroblastoma rat cells were cultured in $75 \mathrm{~cm}^{2}$ flask, in Dulbecco's Modified Eagle Medium (DMEM) supplemented with L-glutamine $(2 \mathrm{mM})$, penicillin $100 \mathrm{IU}$ $\mathrm{ml}-1$, streptomycin (100 mg l-1) and 10\% fetal bovine serum (FBS), and at $37^{\circ} \mathrm{C}$ in a $5 \% \quad \mathrm{CO}_{2}$ humidified chamber.

Cells were seed on glass coverslips placed in a 6 multiwell and then treated with cisplatin at $40 \mu \mathrm{M}$ or Pt(IV)AcPOA at $4 \mu \mathrm{M}$ for $48 \mathrm{~h}$ at continuous exposure. These concentrations for cisplatin and Pt(IV)Ac-POA were based on previous works and obtained by $\mathrm{IC}_{50}$ analysis, respectively [11,12].

After treatment with cisplatin $(40 \mu \mathrm{M})$ and Pt(IV)AcPOA $(4 \mu \mathrm{M})$ for $48 \mathrm{~h}$ at continuous exposure an immunohistochemistry detection for Bax, Bcl-2 and AIF was performed, associated with a labelling for the mitochondria and a counterstained with Hoechst 33258 for the nuclei (Table 1). Images were obtained by an Olympus BX51 microscope equipped with a 100-W mercury lamp and used under the following conditions: 330-385 $\mathrm{nm}$ excitation filter (excf), $400 \mathrm{~nm}$ dichroic mirror (dm) and $420 \mathrm{~nm}$ barrier filter (bf) for Hoechst 33258; 450-480 nm excf, $500 \mathrm{~nm} \mathrm{dm}$ and $515 \mathrm{~nm}$ bf for the fluorescence of Alexa 488; $540 \mathrm{~nm}$ excf, $580 \mathrm{~nm} \mathrm{dm}$ and $620 \mathrm{~nm}$ bf for Alexa 594. Images were then recorded with an Olympus MagniFire camera system and processed with the Olympus Cell F software.

\begin{tabular}{|c|c|c|c|c|}
\hline Antigen & Primary antibody & Dilution in PBS & Secondary antibody & Dilution in PBS \\
\hline Bax & $\begin{array}{c}\text { Polyclonal rabbit (Santa Cruz } \\
\text { Biotechnology) }\end{array}$ & $1: 200$ & $\begin{array}{c}\text { Anti-rabbit IgG1 Alexa Fluor 594 } \\
\text { (Molecular Probes, Invitrogen) }\end{array}$ & $1: 200$ \\
\hline Bcl-2 & $\begin{array}{c}\text { Polyclonal rabbit (Santa Cruz } \\
\text { Biotechnology) }\end{array}$ & $1: 200$ & $\begin{array}{c}\text { Anti- rabbit IgG1 Alexa Fluor 594 } \\
\text { (Molecular Probes, Invitrogen) }\end{array}$ & $1: 200$ \\
\hline AIF & $\begin{array}{c}\text { Polyclonal rabbit (Cell Signaling } \\
\text { Technology) }\end{array}$ & $1: 200$ & $\begin{array}{c}\text { Anti- rabbit IgG1 Alexa Fluor 594 } \\
\text { (Molecular Probes, Invitrogen) }\end{array}$ & $1: 200$ \\
\hline Mitochondria & Human autoimmune serum a & $1: 200$ & $\begin{array}{c}\text { Anti-human IgG1 Alexa Fluor 488 } \\
\text { (Molecular Probes, Invitrogen) }\end{array}$ & $1: 200$ \\
\hline
\end{tabular}

Table 1: Primary and secondary antibodies used for immunocytochemical reactions at fluorescence microscope Rangone, et al. [12]. 


\section{Journal of Embryology \& Stem Cell Research}

Differences between control and treated samples were analysed, evaluating three independent experiments for each condition and protein analysed. The percentage of mean fluorescence intensity was calculated with ImageJ software and the obtained scores were expressed as the mean \pm SEM (standard error of mean). Data differences were analysed for statistical significance by one-way ANOVA and post hoc Bonferroni's test (software package GraphPad Prism Inc.). $\rho$ values ranging from $<0.001$ to $<$ 0.05 were considered statistically significant.

\section{Results}

To analyse the activation of cell death pathways, following treatment with cisplatin at $40 \mu \mathrm{M}$ or Pt(IV)Ac-
POA $4 \mu \mathrm{M}$, immunocytochemical reactions were conducted to put in evidence the proteins involved in these processes: Bax, Bcl-2 and AIF.

After immunolabelling, in the B50 control cells, Bax (red fluorescence) was located in the cytoplasm and not localized with the mitochondria represented by green fluorescence (Figure 1). On the contrary, after both treatments, the Bax-immunolabelling was distributed nearby the mitochondria, where a clear colocalization of the Bax fluorescence with the organelles was observed. Semi quantitative analysis of the immunofluorescence intensity of Bax, showed an increased intensity in cells treated with $\mathrm{Pt}(\mathrm{IV}) \mathrm{Ac}-\mathrm{POA}$, compared to the control and treated-cells, in particular in cells with apoptotic nucleus.

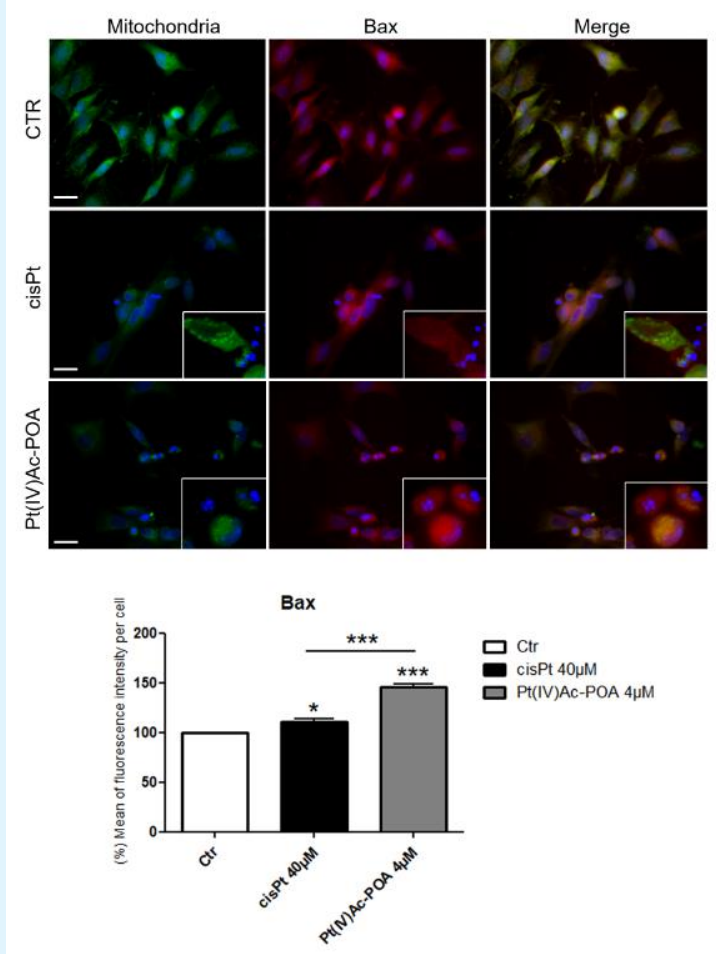

Figure 1: Bax protein analysis. B50 cells in control condition and after treatment with cisplatin $40 \mu \mathrm{M}$ or Pt(IV)AcPOA $4 \mu \mathrm{M}$. Green fluorescence for mitochondria, red for Bax and Hoechst 33258 counterstaining for the nuclei in blue. Inserts show detail of immunolobelling in control and in apoptotic cells. Bars: $40 \mu$ m. Histograms showed the percentage of mean fluorescence intensity per cells normalised to control (Ctr). Statistical analysis: number of observations per control and treated samples, and cisplatin vs Pt(IV)Ac-POA: ${ }^{*} \rho<0.05,{ }^{* * *} \rho<0.001$.

Images reported in Figure 2 show a double immunolabeling for Bcl-2, marked in red, and the mitochondria, green fluorescence. In B50 control cells, immunolabelling for Bcl-2 protein was distributed in the cytoplasm and any colocalization with the mitochondria fluorescence was detectable. After treatment with cisplatin at $40 \mu \mathrm{M}$ and $\mathrm{Pt}(\mathrm{IV}) \mathrm{Ac}-\mathrm{POA}$ at $4 \mu \mathrm{M}$, respectively, Bcl-2 translocated to the mitochondria in early apoptosis, while its expression decreases, resulting almost absent in late apoptosis, as can be seen in the inserts. To note, in 


\section{Journal of Embryology \& Stem Cell Research}

cells exposed to treatment with cisplatin, an increasing in Bcl-2 fluorescence was observed and corroborated by semi quantitative analysis. In fact, B50 cells that show a control-like phenotype after treatment show an increase in fluorescence for the protein under examination.

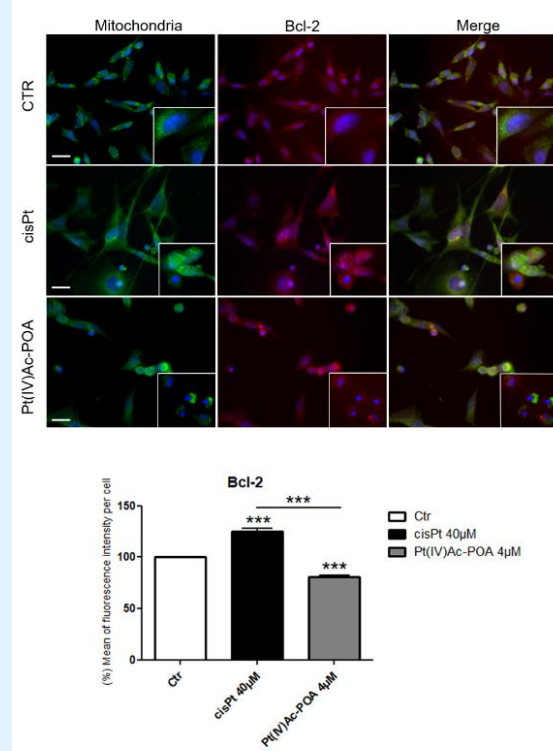

Figure 2: Bcl-2 protein analysis. B50 cells in control condition and after treatment with cisplatin $40 \mu \mathrm{M}$ or Pt(IV)AcPOA $4 \mu \mathrm{M}$. Green fluorescence for mitochondria, red for Bcl-2 and Hoechst 33258 counterstaining for the nuclei in blue. Inserts show detail of immunolobelling in control and in apoptotic cells. Bars: $40 \mu \mathrm{m}$. Histograms showed the percentage of mean fluorescence intensity per cells normalised to control (Ctr). Statistical analysis: number of observations per control and treated samples, and cisplatin vs Pt(IV)Ac-POA: ${ }^{* * *} \rho<0.001$.

Immunolabel for AIF, red fluorescence, revelled that in B50 control cells, AIF fluorescence localizes with the mitochondria (green fluorescence) (Figure 3). After treatment with cisplatin at $40 \mu \mathrm{M}$ or Pt(IV)Ac-POA at 4 $\mu \mathrm{M}$, the fluorescence related to AIF was no longer associated with labelling for mitochondria, but was found at the nuclear level which, following treatment, appears fragmented and with apoptotic features.

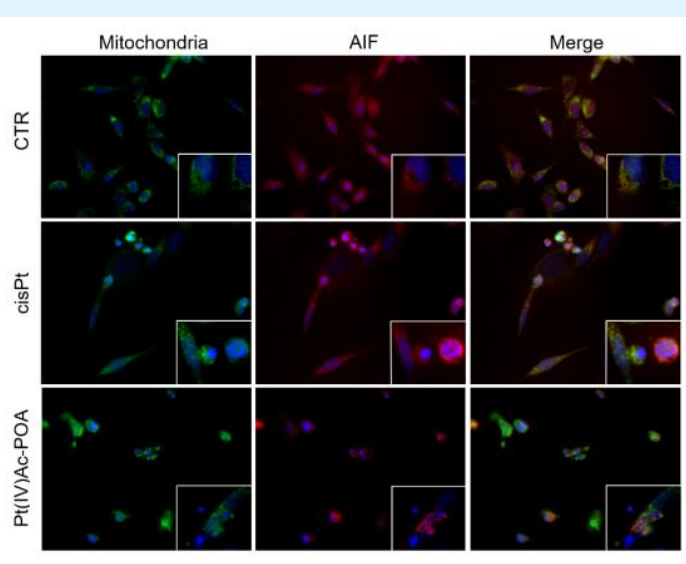

Figure 3: AIF protein analysis. B50 cells in control condition and after treatment with cisplatin $40 \mu \mathrm{M}$ or Pt(IV)AcPOA $4 \mu \mathrm{M}$. Green fluorescence for mitochondria, red for AIF and Hoechst 33258 counterstaining for the nuclei in blue. Inserts show detail of immunolobelling in control and in apoptotic cells. Magnifications: 40x, inserts: 100x. 


\section{Journal of Embryology \& Stem Cell Research}

At last, in each condition analysed, a severe alteration in mitochondria structure were observed after both treatments compared to control. Indeed, in control cells mitochondria were characterized by a fusiform aspect and a homogeneous localization in the cytoplasm. Following treatment with cisplatin or Pt(IV)Ac-POA, the mitochondria lose their structure, appeared smaller and more rounded, clustering in dense masses around cells nuclei. A strong reduction in the number and in the cell size were evident after exposure Pt(IV)Ac-POA compared to control and cisplatin-treated cells. In particular, after cisplatin exposure some cells showed a treatmentresistant phenotype, the mitochondria maintain morphology comparable to that found in control cells and proteins of interest remained in the physiological compartment. This data was not observed after treatment with Pt(IV)Ac-POA or at least was detected in a minor way compared to cisplatin, suggesting a greater proapoptotic effect of the new compound.

\section{Conclusion}

Results presented in this work show that the prodrug can activate both the apoptotic pattern in B50 cell lines: the intrinsic apoptotic pathway and the independent caspase pattern. After treatment with Pt(IV)Ac-POA a morphological change in mitochondria, which undergo fission, were observed associated to i) an increase in the Bax protein level, ii) the translocation of AIF from the mitochondria to the nucleus and iii) a decrease in Bcl-2. Quantified values, which are statistically significantly, corroborates the greater effect of the new compound, active already at $4 \mu \mathrm{M}$, than cisplatin $(40 \mu \mathrm{M})$. After Pt(IV)Ac-POA $4 \mu \mathrm{M}$ treatment, as had already been demonstrated by Rangone, et al. [12], the number of cells dying due to apoptosis or autophagy is also greater, compared to the standard treatment with cisplatin, where, the cells that do not show an apoptotic morphology and cells are characterized by an increasing in Bcl-2 protein expression, suggesting a cisplatin-chemo resistance.

\section{Conflict of Interest}

The authors declare that they have no conflict of interest

\section{Acknowledgment}

This research was supported by Italian Ministry of Education, University and Research (MIUR): Dipartimenti di Eccellenza Program (2018-2022) - Dept. of Biology and Biotechnology “L. Spallanzani”, University of Pavia

\section{Funding Information}

This research is supported by the University of Pavia: Fondi Ricerca Giovani (FRG 2018) and by the Compagnia di San Paolo: research project "BIPLANES". We are indebted to the Inter-University Consortium for Research on the Chemistry of Metals in Biological Systems (CIRCMSB, Bari) and UE COST CM1105 Action "Functional metal complexes that bind to biomolecules" for stimulating discussions during the group meetings and short-term missions.

This research was supported by Italian Ministry of Education, University and Research (MIUR): Dipartimenti di Eccellenza Program (2018-2022) - Dept. of Biology and Biotechnology “L. Spallanzani”, University of Pavia.

\section{References}

1. Boulikas T, Pantos A, Bellis E, Christofis P (2007) Designing platinum compounds in cancer: structures and mechanism. Cancer Ther 5: 537-583.

2. Yanagisawa R, Kubota N, Hidaka E, Sakashita K, Tanaka M, et al. (2018) Cisplatin-induced nephrotoxicity in patients with advanced Neuroblastoma. Pediatr Blood Cancer 65(9): e27253.

3. Wei M, Yuan X (2019) Cisplatin-induced ototoxicity in children with solid tumor. J Pediatr Hematol Oncol 41(2): e97-e100.

4. Aveic S, Corallo D, Porcù E, Pantile M, Boso D, et al. (2018) TP-0903 inhibits neuroblastoma cell growth and enhances the sensitivity to conventional chemotherapy. Eur J Pharmacol 818: 435-448.

5. Graf N, Lippard SJ (2012) Redox activation of metalbased prodrugs as a strategy for drug delivery. Adv Drug Deliv Rev 64(11): 993-1004.

6. Wexselblatt E, Gibson D (2012) What do we know about the reduction of $\mathrm{Pt}(\mathrm{IV})$ pro-drugs? J Inorg Biochem 117: 220-229.

7. Johnstone TC, Suntharalingam K, Lippard SJ (2016) The next generation of platinum drugs: targeted Pt(II) agents, nanoparticle delivery, and $\mathrm{Pt}(\mathrm{IV})$ prodrugs. Chem Rev 116: 3436-3486.

8. Li Y, Seto E (2016) HDACs and HDAC Inhibitors in cancer development and therapy. Cold Spring Harb Perspect Med 6(10): 026831. 


\section{Journal of Embryology \& Stem Cell Research}

9. Novohradsky V, Zerzankova L, Stepankova J, Vrana O, Raveendran R, et al. (2015) New insights into the molecular and epigenetic effects of antitumor Pt(IV)valproic acid conjugates in human ovarian cancer cells. Biochem Pharmacol 95(3): 133-144.

10. Witt O, Deubzer HE, Lodrini M, Milde T, Oehme I (2009) Targeting histone deacetylases in neuroblastoma. Curr Pharm Des 15(4): 436-447.
11. Santin G, Scietti L, Veneroni P, Barni S, Bernocchi G, et al. (2012) Effects of Cisplatin in neuroblastoma rat cells: damage to cellular organelles. Int J Cell Biol 2012: 424072 .

12. Rangone B, Ferrari B, Astesana V, Masiello I, Veneroni $P$, et al. (2018) A new platinum-based prodrug candidate: Its anticancer effects in B50 neuroblastoma rat cells. Life Sci 210: 166-176. 